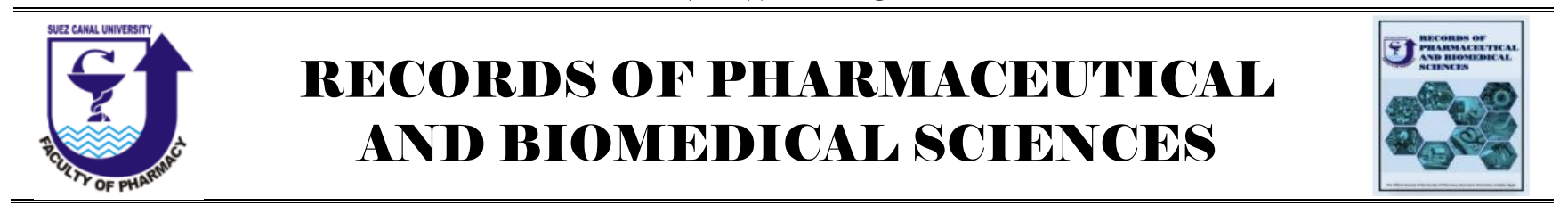

\title{
Novel Adipokines and the Risk of Obesity-Related Breast Cancer
}

\author{
Dalia M. Asal ${ }^{\text {**, Noha M. Mesbah }}{ }^{\mathrm{a}}$, Dina M. Abo-Elmatty ${ }^{\mathrm{a}}$, Hamada Fathy ${ }^{\mathrm{b}}$, Asmaa R. Abdel-Hamed ${ }^{\mathrm{a}}$ \\ ${ }^{a}$ Department of Biochemistry, Faculty of Pharmacy, Suez Canal University, Ismailia, Egypt, ${ }^{b}$ Surgical \\ Oncology Unit, Department of Surgery, Faculty of Medicine, Suez Canal University, Ismailia, Egypt
}

\section{Received: 20. 10. 2021}

Revised: 10. 11. 2021

Accepted: 13. 11. 2021

*Correspondence Author:

Tel: $+2-01284831384$

E-mail address:

daliaasal_staff@pharm.suez.edu.eg

\begin{abstract}
Breast cancer is one of the most prevalent malignant tumors around the world, and it is the leading cause of cancer death in women. Obesity is linked to an increased risk of breast cancer, especially in postmenopausal women. The prominence of the triad of overweight/obesity, insulin resistance, and adipokines in cancer has been highlighted in recent studies. Adipose tissue has a vital endocrine role, secreting numerous biochemicals that affect human physiology. Obesity impairs this role, resulting in alterations in adipokine release, which contribute to the development of carcinogenesis. Among these adipokines are vaspin and omentin-1. Vaspin is a serine protease inhibitor produced from visceral adipose tissue that is well recognized for its insulin-sensitizing properties and modulatory role in glucose tolerance. Vaspin also exhibits antiinflammatory effects. Omentin-1 improves insulin sensitivity and has been linked to a lower risk of obesity, along with a potential role as a tumor suppressor in cancers such as prostate, liver, colorectal, and pancreatic cancer. To the extent of our knowledge, there are few studies that analyze the genetic association of vaspin and omentin-1 with breast cancer risk.
\end{abstract}

Keywords: Breast cancer; Adipokines; Vaspin, Omentin; Insulin resistance; Obesity.

\section{Introduction}

Breast cancer has consistently been ranked among the top women's cancers for many years, in both aspects of incidence and mortality (Naeem et al., 2019). According to GLOBOCAN (Global Cancer Observatory) data, breast cancer accounted for $16.4 \%$ of all new cancer cases and $32.4 \%$ of cancer cases among Egyptian women in 2020 (https://gco.iarc.fr/today/).

Obesity is now identified as a risk factor for the development of a wide range of comorbid diseases, including type 2 diabetes, cardiovascular disease, and a rising number of cancers (Fang et al., 2018; Ludwig et al., 2018).

Obesity and excess adipocyte accumulation are implicated in the development of breast cancer risk, particularly in post-menopausal women (Blucher et al., 2017). In obesity, adipocytes and breast cancer cells interact at the molecular level via several circulating molecules. Obese adipocytes become hypertrophic, secreting more bioactive lipids, pro-inflammatory cytokines, and adipokines (Iyengar et al., 2013; Lengyel et al., 2018). These biochemicals have been associated with chronic low-grade inflammation, 
which has been connected to cancer progression (Iyengar et al., 2013). Recent studies have highlighted the importance of the triad of obesity, insulin resistance, and adipokines in cancer (Spyrou et al., 2018). The altered generation of adipokines by obese adipose tissue is involved in the development of cancer (Cabia et al., 2016). These adipokines include vaspin and omentin-1 (Spyrou et al., 2018).

\section{Breast cancer pathogenesis}

Breast cancer tends to start as ductal hyperproliferation and progresses to benign tumors or even metastatic carcinomas due to long-term stimulation by respective carcinogenic factors. The tumor microenvironment is a complex ecosystem composed of infiltrating immune cells, mesenchymal support cells, and matrix components that play critical roles in the onset and progression of breast cancer (Sun et al., 2017). Adipocytes are the primary cellular components of the breast cancer microenvironment, and emerging evidence suggests that adipocytes promote tumorigenesis through mutual and interactive communication between cancer cells and adipocytes (Wu et al., 2019).

\subsection{Breast cancer risk factors}

Breast cancer risk factors include both genetic and non-genetic factors. Commonly, environmental factors combined with genetic factors have an impact on breast cancer development (De Silva et al., 2019). A variety of risk factors for breast cancer, including aging and female sex, have been well-established by epidemiologic studies conducted to date. Non-modifiable factors like race, ethnicity, and genetics, and also modifiable factors such as diet, physical inactivity, BMI, exogenous hormones, and certain female reproductive factors, are among these risk factors (Ahmad, 2019).

Obesity comorbidities such as cardiovascular disease, diabetes, and cancer have been linked to insulin resistance and hyperinsulinemia. Insulin has anabolic effects on cellular metabolism, and human cancer cells overexpress the insulin receptor (Klement et al., 2016).

\subsection{Adipocyte biology in breast cancer}

Normal breast tissue and breast cancer both have an interaction between epithelial cells and adipocytes, and this close interaction occurs throughout the cancer process: tumor initiation, progression, invasion, and metastasis as explained in Figure 1 (Choi et al., 2018).

Several studies have shown that adipocytes, which are major components of the stromal environment of mammary tumors, have tumor-promoting effects on breast cancer cells at the molecular level. Adipocytes release biologically active lipids, hormones, adipokines, and proteases/protease inhibitors, preparing breast cancer cells to become more aggressive. Subsequently, adipocyte lipolysis is altered by breast cancer cells, leading to the formation of cancer-associated adipocytes (CAAs). As a result, the elevated secretion of free fatty acids (FFA), inflammatory cytokines, and proteases from CAAs promotes the progression of breast cancer (Wu et al., 2019). The resulting increased secretion of FFA, insulin-like growth factor-1 (IGF-1), insulin, inflammatory cytokines, and altered secretion of adipokines enhances the cancerpromoting effects of adipocytes (Figure 2) (Blucher et al., 2017; Argolo et al., 2018).

\section{Obesity}

Obesity is a chronic low-grade inflammation characterized by abnormal excess fat deposition in adipocytes. The World Health Organization (WHO) and the National Institutes of Health (NIH) refer to body weight as a body mass index (BMI, defined as weight $(\mathrm{kg}) /$ height $\left(\mathrm{m}^{2}\right)$. Overweight individuals have a BMI of $25-29 \mathrm{~kg} / \mathrm{m}^{2}$, while obese individuals have a BMI $\geq 30 \mathrm{~kg} / \mathrm{m}^{2}(30.0$ 34.9 , grade I; $35.0-39.9$, grade II; and $\geq 40$, grade III).

Obese postmenopausal women are at a higher risk of developing hormone-sensitive tumors due to high levels of estrogen in serum and peripheral site production of this hormone (Mohanty et al., 2021). Likewise, adiposopathy (excess adiposity) in postmenopausal females was linked to an increased risk of breast cancer, particularly hormone-dependent estrogen/progesterone receptor positive (ER/PR +ve) breast cancer (Iyengar et al., 2019).

\subsection{Main mechanisms linking obesity and breast cancer}

There are several mechanisms that contribute to the connection between breast cancer and obesity, including chronic subclinical inflammation, sex hormone deregulation, insulin/IGF-1 pathways, and 

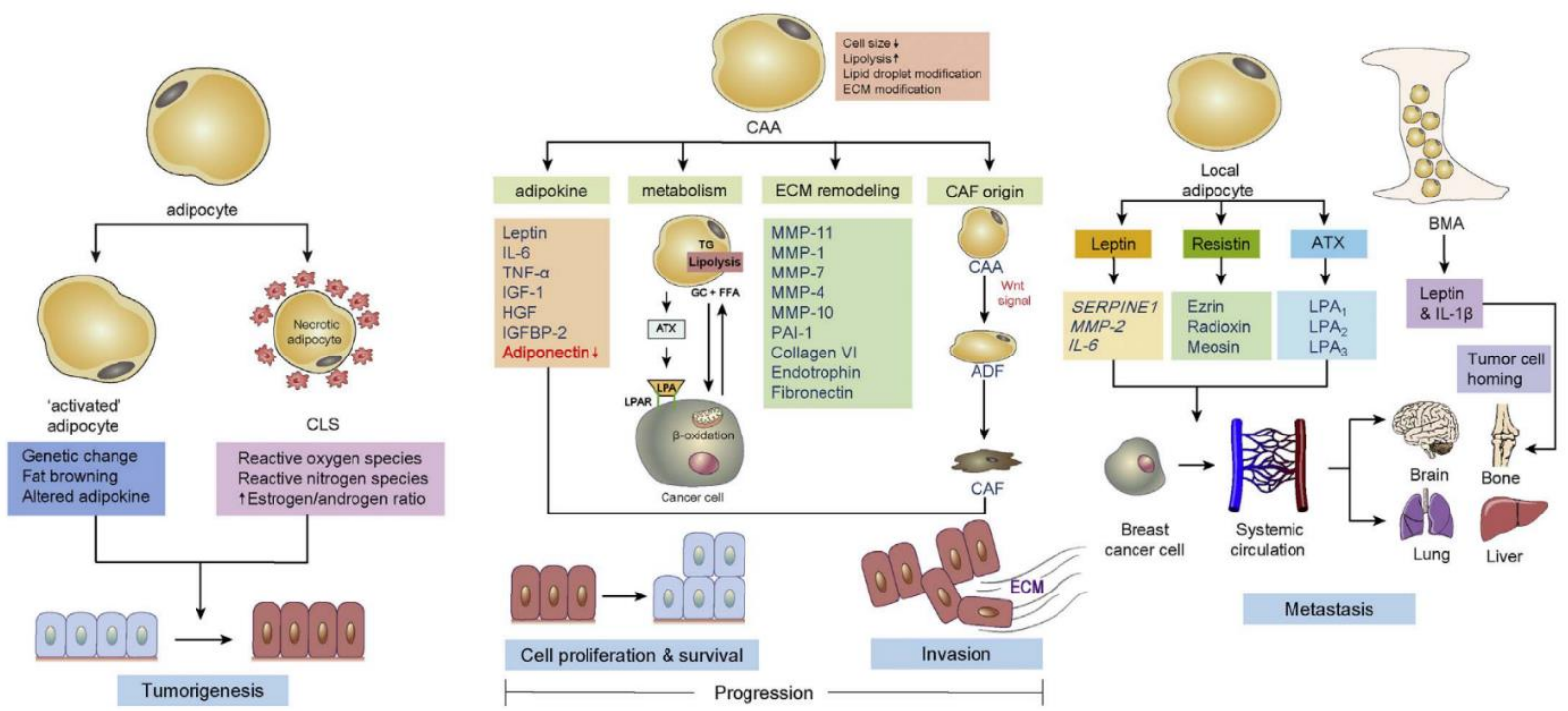

Figure 1 : Role of adipocyte in breast cancer (Choi et al., 2018). CLS; crown like structure, CAA; cancer-associated adipocyte, ATX; autotoxin, LPA; lysophosphatidate, LPAR; lysophosphatidate receptor, ECM; extracellular matrix, TG; triglyceride, GC; glycerol, FFA; free fatty acid, ADF; adipocyte-derived fibroblast, CAF; cancer-associated fibroblast, BMA; bone marrow adipocyte; IL; interleukin, IGF; Insulin-like growth factor, HGF; hepatocyte growth factor, MMP; matrix metalloproteinase, PAI; plasminogen activator inhibitor, IGFBP-2; Insulin-like growth factor (IGF) binding protein-2.

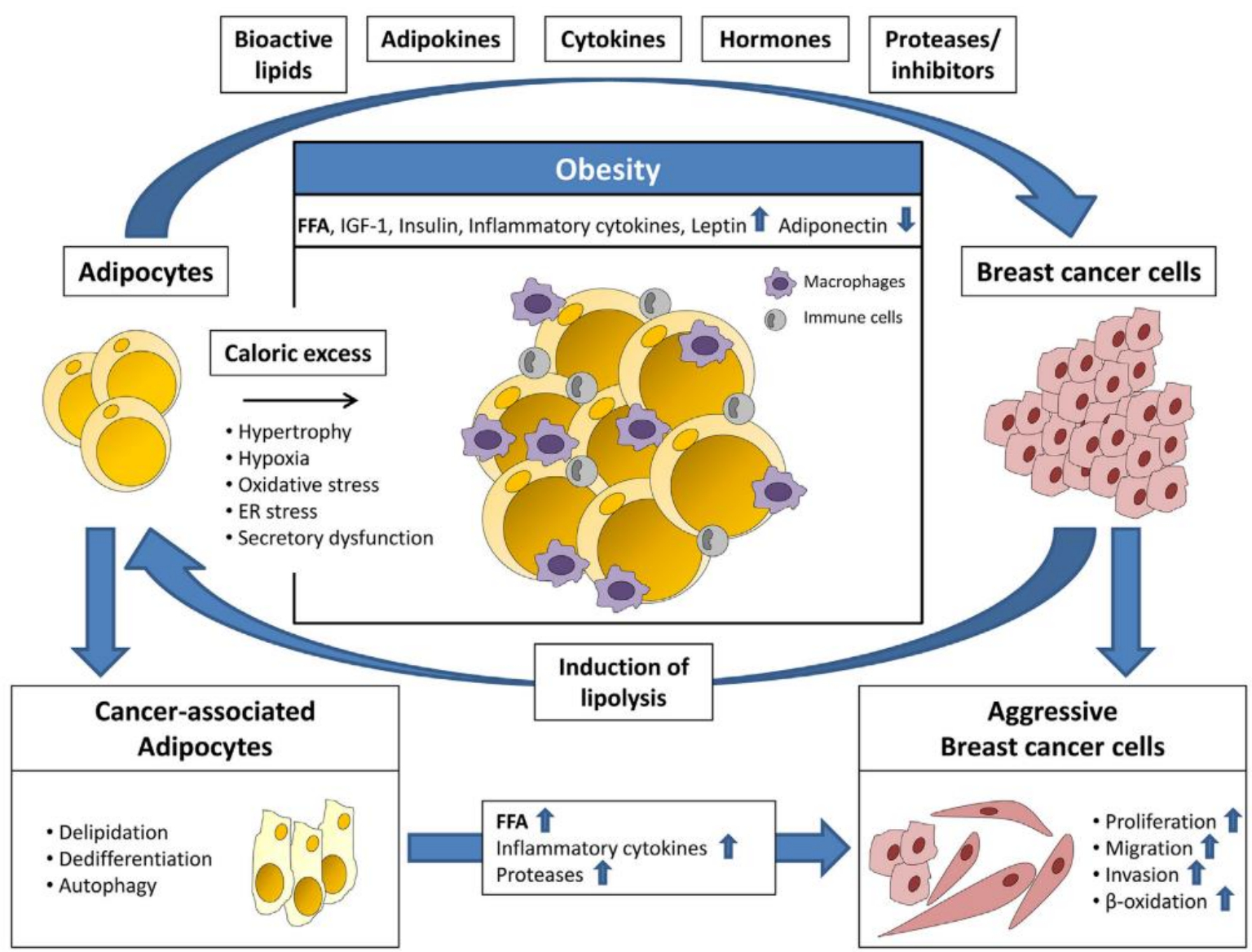

Figure 2 : Molecular interactions between adipocytes and breast cancer cells (Blucher et al., 2017).FFA; free fatty acids, IGF-1; insulin-like growth factor-1. 
adipokine secretion (Sanchez-Jimenez et al., 2019).

\section{a) Chronic inflammation}

Adipose chronic inflammation has been reported to increase cancer cell survival and development, promote angiogenesis, and metastasis (Iyengar et al., 2016). Obesity is also associated with higher levels of pro-inflammatory mediators such as interleukin-6 (IL-6), tumor necrosis factor alpha (TNF- $\alpha$ ), matrix metalloproteinase-9 (MMP-9) and interleukin-1 (IL-1), all of which have been linked to cancer (Divella et al., 2016).

\section{b) Sex hormone deregulation}

After menopause, adipose tissue becomes the main source of estrogens by aromatase from stromal cells. Aromatase activity among obese women is high, leading to increased production of estrogens and relatively high levels in plasma, which is linked to an increase in breast cancer incidence (Feola et al., 2017). Factors produced during inflammation and the paracrine loop may induce the aromatase enzyme, with the consequent stimulation of ER-positive breast cancer cell growth through locally produced estrogens. As a result, it seems that obesity, inflammation, and hormonereceptor positive cancer are all linked (Crespi et al., 2016).

\section{c) Hyperinsulinemia}

Hyperinsulinemia promotes cancer development both directly and indirectly through the decrease in circulating levels of IGF-1 binding proteins, which improves the bioavailability of IGF-1. High IGF-1 has been associated with an increased risk of breast cancer in both premenopausal and postmenopausal women (Laudisio et al., 2018). Hyperglycemia also appears to increase the risk of breast cancer in premalignant cells and increase the progression of cancer in malignant epithelial cells through leptin/IGF1 signaling (Lopez et al., 2013; SánchezJiménez et al., 2019).

\section{d) Adipokines}

Adipocytes are the main cellular components of adipose tissue. Adiposopathy, which occurs in obesity, can secrete excessive biomolecules, including adipokines, that are linked to metabolic dysfunction, insulin resistance, and inferior outcomes in cancer treatment, as well as creating an environment that promotes cancer invasion and metastasis (Picon-Ruiz et al., 2017).

\section{Adipokines}

Adipokines are bioactive hormones generated and released by adipose tissue. These biochemicals perform multiple functions, involving regulation of metabolism and caloric intake, along with angiogenesis and cell proliferation (Hursting et al., 2012; Kojta et al., 2020). The production and secretion of various adipokines are modulated by several stimuli, including insulin, estrogens, and inflammatory mediators (Goodwin et al., 2015; Cici et al., 2021). In the context of obesity, adipokine levels are commonly disrupted (Zorena et al., 2020) and this dysregulation has been implicated in cancer development and metastasis (Spyrou et al., 2018).

More than 15 adipokines have been linked to cancer, and the number is continually growing (Dalamaga et al., 2017). While several circulating pro-inflammatory adipokines, including leptin, TNF- $\alpha$, IL-6, resistin, and extracellular Nampt (eNampt), are increased in tumors, some adipokines, such as adiponectin and omentin-1, are lowered in tumors and are thought to be anticarcinogenic (Reizes et al., 2016; Parida et al., 2019).

\subsection{Adipokines in breast cancer promotion}

The serum concentration of adipokines and underlying mechanisms in breast cancer are shown in (Figure 3) (Christodoulatos et al., 2019). The figure presents the variation in circulating adipokine levels, showing that some adipokines, such as leptin, resistin, visfatin, and chemerin, are raised in breast cancer while others, such as adiponectin and irisin, are lowered in breast cancer, which might be considered protective against breast carcinogenesis. Through estrogen receptor and aromatase expression modulation, changes in the bioavailability of various growth factors, and inflammation, adipokines may play a role in breast cancer development, proliferation, migration, and neoangiogenesis. The following evidence supports the link between adipokines and breast cancer risk and progression: alterations in plasma concentrations in breast cancer patients, their link to advanced stage and prognosis in breast cancer (prognostic biomarkers), their differential expression in benign and malignant breast tissues, their overexpression in breast tumor tissues, their relationship with cancer therapy resistance (predictive biomarkers), their link with in vivo and 


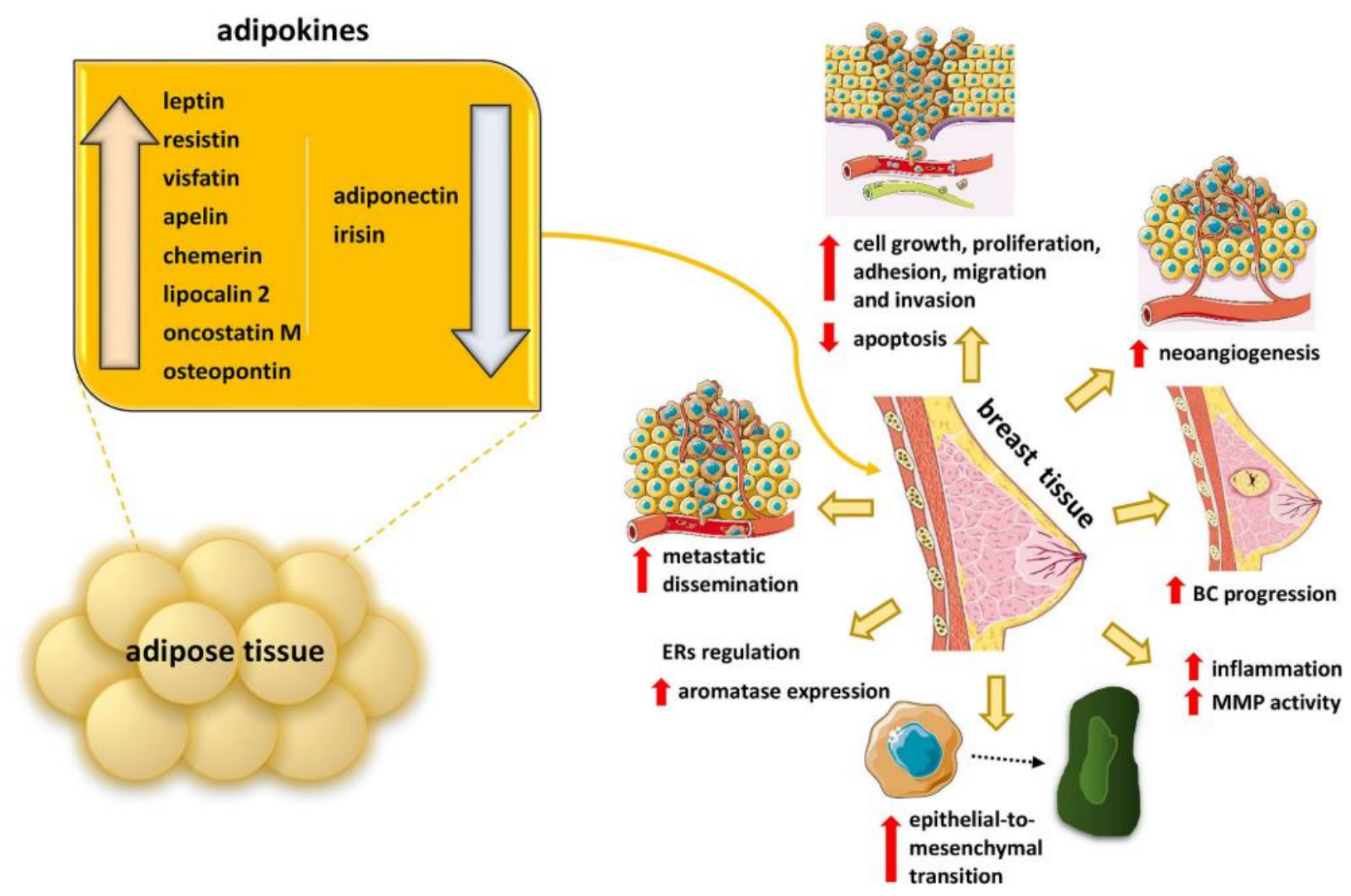

Figure 3: Adipokines serum concentrations and underlying mechanisms in breast cancer (Christodoulatos et al., 2019).

in vitro models of breast cancer, and the correlation of genetic variants in adipokines and their receptor genes with breast cancer (Spyrou et al., 2018).

\section{a) Classic adipokines}

Classic adipokines that were previously found, such as leptin and adiponectin, have been widely investigated in cancer. Well-established links between classic and novel adipocytokines and cancer risk and progression include alterations in plasma or serum concentrations in cancer patients; differential expression in malignant and benign tissues; and overexpression in tumor tissues (Spyrou et al., 2018).

Regarding adiponectin and breast cancer, adiponectin serum levels show an inverse association with adipose tissue mass and have been shown to exert protective roles against the development of obesity-related disorders, including cancer (Dalamaga et al., 2012). In addition, adiponectin exhibits anti-proliferative, antimigratory, and pro-apoptotic actions (Spyrou et al., 2018). Serum adiponectin levels were lower in $B C$ patients irrespective of menopausal status (Gu et al., 2018).
Interestingly, other meta-analyses have found a significant association between adiponectin levels and postmenopausal breast cancer patients but not premenopausal (Ye et al., 2014).

Concerning leptin and breast cancer, the oncogenic mechanism of leptin in breast tissue involves the stimulation of JAK/STAT3 and PI3K pathways. Leptin can inhibit apoptosis of breast cancer cells by favoring the expression of anti-apoptotic genes (bcl-xL, bax) and induce angiogenesis by stimulation of VEGF production (Christodoulatos et al., 2019). Leptin has a remarkable interaction with estrogen signaling. Leptin can enhance estrogen signaling via three different pathways, including upregulation of aromatase, direct activation of the estrogen receptor (Era), and suppression of tumor protein p53 (Christodoulatos et al., 2019).

\section{b) Novel adipokines}

There are several novel adipokines such as resistin, chemerin, omentin, vaspin, nesfatin, eNampt/visfatin, and osteopontin that are involved in a wide range of physiological and pathological processes. Alterations in serum levels of classic and novel adipocytokines in cancer are illustrated 
in Figure 4 (Spyrou et al., 2018).

Chemerin expression is decreased in breast cancer patients compared to normal controls, and it seems to be associated with a poor survival outcome (EISagheer et al., 2018). There is conflicting evidence about chemerin's clinical value as a prognostic factor in breast cancer. Chemerin expression in breast tissue was associated with a poor prognosis as well as adverse clinical and pathological characteristics (El-Sagheer et al., 2018).

\subsection{Vaspin}

Vaspin is a novel adipose tissue-derived serpin family member. Interestingly, it has been postulated that vaspin could be a compensating adipocytokine that enhances insulin sensitivity (Hida et al., 2005). In hyperglycemia, vaspin inhibits insulin receptor substrate-2 (IRS2) function by reducing phosphorylation of the insulin receptor and its products. This is a protective mechanism against tissue hyperinsulinemia (Li et al., 2011).
Heiker in 2014 described vaspin's antiinflammatory role in endothelial cells, and its involvement in inhibiting smooth muscle cell migration by reducing reactive oxygen species formation as shown in (Figure 5) (Heiker, 2014). Vaspin suppresses tumor necrosis factor (TNF), platelet derived growth factor (PDGF), methylglyoxal, which is one of the reactive intermediates produced during the metabolic reprogramming of cancer cells and is considered as a pro-cancer metabolite and novel biomarker in cancer (Leone et al., 2021), and high glucoseinduced reactive oxygen species (ROS) generation, and hence decreases cell apoptosis (lower caspase3 activity), monocyte adherence, and cytoskeletal remodeling and movement. Vaspin raises intracellular NO via STAT3-induced dimethylaminohydrolase (DDAH) production as well as Akt activation.

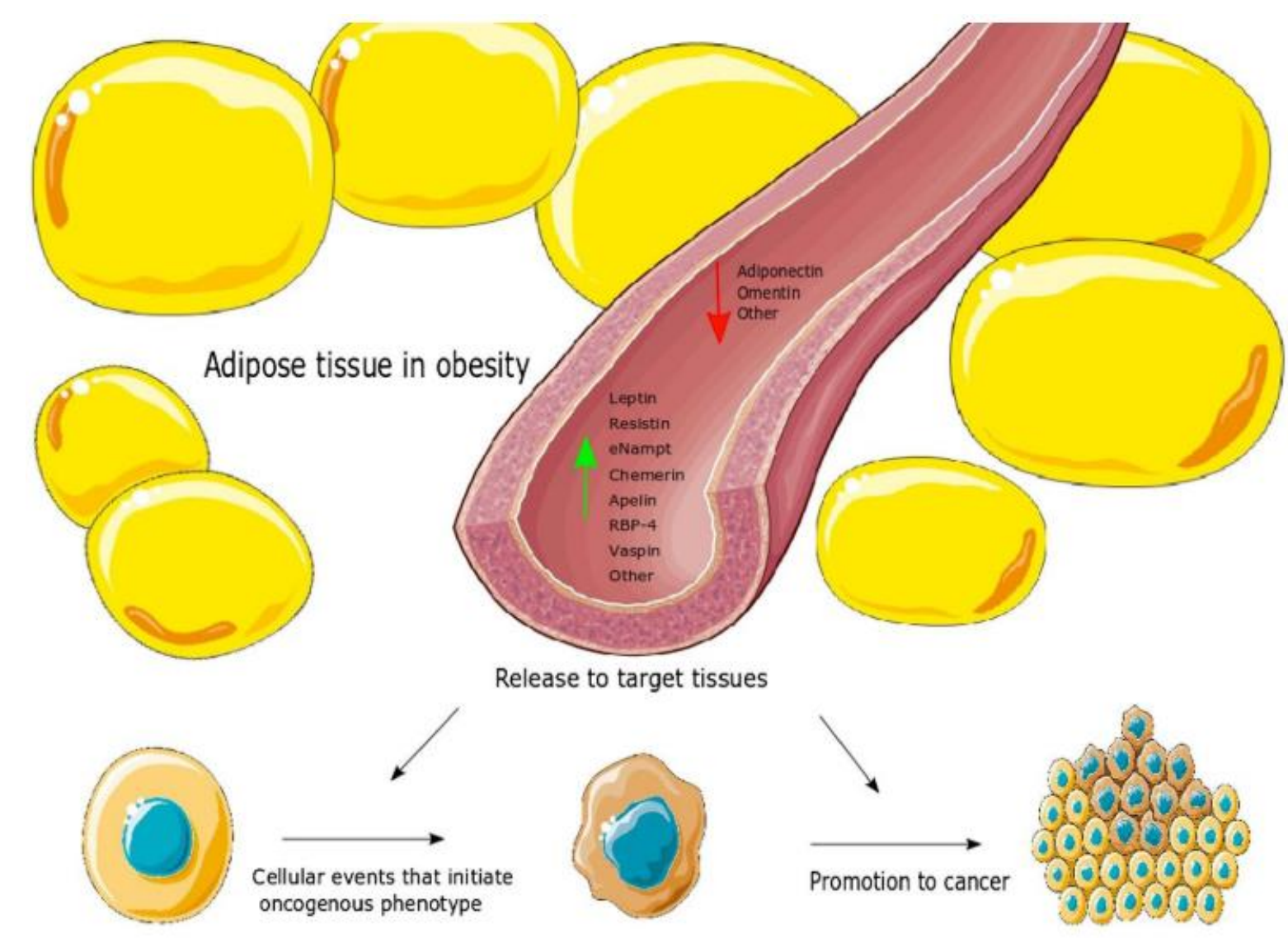

Figure 4: Alterations in serum levels of classic and novel adipokines in cancer (Spyrou et al., 2018). 


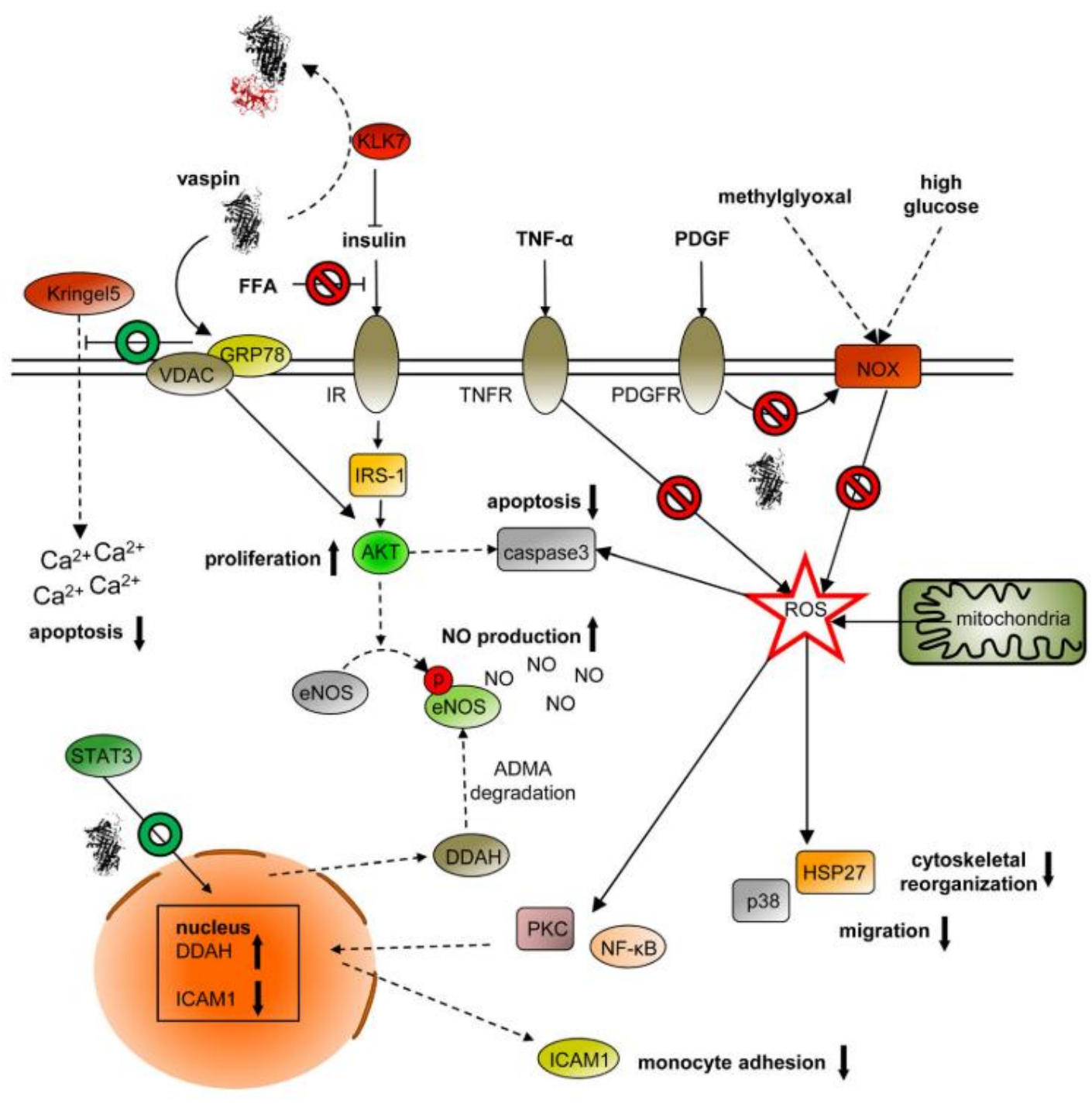

Figure 5: Anti-inflammatory and anti-apoptotic impacts of vaspin (Heiker, 2014). IR; insulin receptor, TNFR; tumor necrosis factor receptor, PDGFR; platelet-derived growth factor receptor, PKC; protein kinase C, VDAC; voltagedependent anion channel, NOX; NADPH oxidase.

Vaspin can be observed in a number of diseases, including diabetes, obesity, metabolic syndrome, polycystic ovarian syndrome (PCOS), and cardiovascular diseases (Wada, 2008; ElMesallamy et al., 2011). Curiously, a reduced vaspin level has been linked to an increased risk of endometrial cancer (Cymbaluk-Ploska et al., 2018), which could support its anti-inflammatory effect. Another study, however, discovered greater levels of vaspin-1 in colorectal cancer patients (Fazeli et al., 2013). As a result, the data linking vaspin to cancer has been muddled by conflicting findings. Further research to determine a clear connection between vaspin and cancer is required (Cabia et al., 2016; Spyrou et al., 2018). The association of vaspin rs2236242 polymorphism has been inspected in recent studies, such as obesity
(Kempf et al., 2010), metabolic syndrome, PCOS (Kohan et al., 2014), and psoriasis (DizenNamdar et al., 2020).

\subsection{Omentin-1}

Omentin-1, formerly known as intelectin-1, is secreted by adipose tissue (Yang et al., 2006). As shown in Figure 6, omentin has been found to play a role in inflammatory reactions and cell differentiation via the AMPK/eNOS signaling pathway, which inhibits JNK activity to repress inflammatory responses and promote cell differentiation (Ohashi et al., 2014). Omentin also induces apoptosis in cells via activating the JAK signaling pathway and increasing P53 expression (Zhang et al., 2013). 


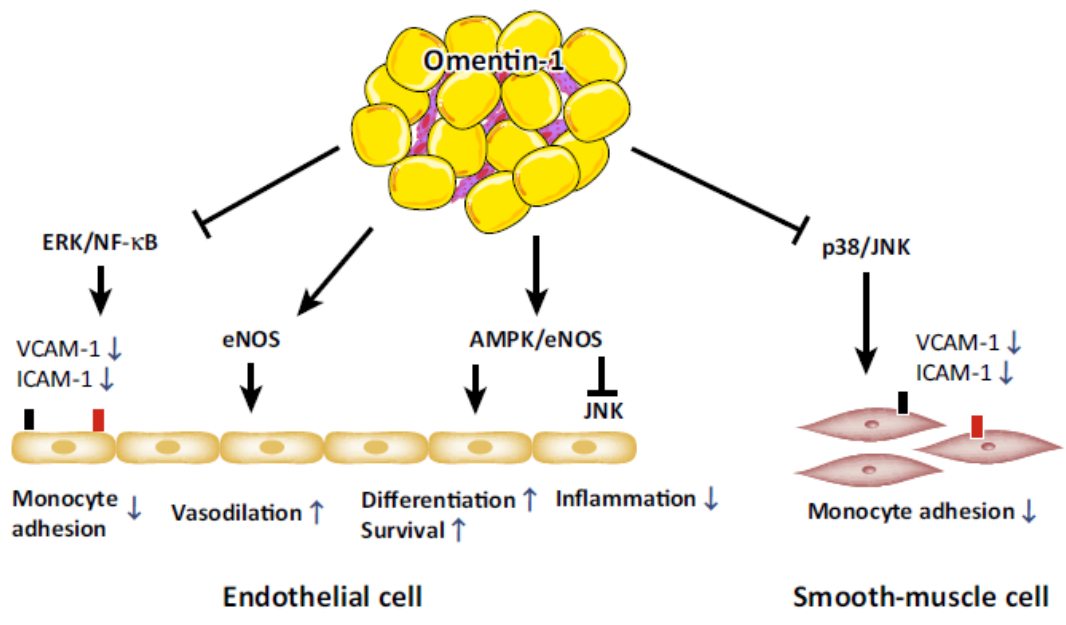

Figure 6: Protective function of omentin-1 in the vasculature (Ohashi et al., 2014). VCAM; vascular cell adhesion molecule, ICAM-1; intercellular cell adhesion molecule-1, ERK; extracellular signal-regulated kinase, NF; nuclear factor, AMPK; AMP activated protein kinase, eNOS; endothelial nitric oxide synthase, JNK; c-Jun N-terminal kinase.

Schäffler et al. identified a single nucleotide polymorphism in exon 4 of the omentin gene for the first time in 2007 and observed that the nucleotide +326 is polymorphic $(\mathrm{A} / \mathrm{T})$. As a result, the codon GAC was substituted with GTC, and the amino acid Asp was changed to $\mathrm{Val}$ at position 109 (Schaffler et al., 2007). The omentin val109Asp polymorphism has been examined in different studies, including psoriasis as a chronic inflammatory disease (Turan et al., 2014), coronary artery disease (Yoruk et al., 2014; Nazar et al., 2017), and breast cancer (Bahadori et al., 2014).

The role of omentin-1 in various malignancies is unclear (Karabulut et al., 2016). According to previous studies, the serum level of omentin-1 is increased in patients with chronic and acute pancreatitis (Karabulut et al., 2016), prostate (Uyeturk et al., 2014), and colorectal cancer (Fazeli et al., 2013). However, its serum level is lowered in individuals with breast cancer (Nourbakhsh et al., 2018; Tahmasebpour et al., 2020), renal cell carcinoma (Shen et al., 2016), lung cancer (Ansari et al., 2018) and endometrial cancer (Holman et al., 2014).

\section{Conclusion}

Obesity and breast cancer are linked in an alarming way, providing a substantial public health risk. Obesity is now recognized as a significant risk factor for the development and progression of breast cancer. Several mechanisms, including inflammatory signals and adipokines, have been proposed to explain this connection. This review outlines the links between obesity and the development of breast cancer, as well as the findings of the relationship between classic and novel adipokines and breast cancer risk.

\section{Conflict of interest}

None of the authors have any conflicts of interest.

\section{References:}

Ahmad, A. 2019. Breast Cancer Metastasis and Drug Resistance: Challenges and Progress, Springer Nature.1152.

Ansari, M. H. K., M. Gholamnejad, K. Meghrazi and H. R. Khalkhali, 2018. Association of circulating omentin-1 level with lung cancer in smokers. Med J Islam Repub Iran, 32 (1), 133.

Argolo, D. F., C. A. Hudis and N. M. Iyengar, 2018. The Impact of Obesity on Breast Cancer. Curr Oncol Rep, 20 (6), 47.

Bahadori, M., L. Kohan, M. Farzan, S. Aliakbari and M. Mohammadian Panah, 2014. An increased risk of breast cancer associated with Val109Asp polymorphism in omentin gene. Int J Biosci, 5 (1), 429-434. 
Blucher, C. and S. C. Stadler, 2017. Obesity and Breast Cancer: Current Insights on the Role of Fatty Acids and Lipid Metabolism in Promoting Breast Cancer Growth and Progression. Front Endocrinol, 8293.

Cabia, B., S. Andrade, M. C. Carreira, F. F. Casanueva and A. B. Crujeiras, 2016. A role for novel adipose tissue-secreted factors in obesityrelated carcinogenesis. Obes Rev, 17 (4), 361-376.

Choi, J., Y. J. Cha and J. S. Koo, 2018. Adipocyte biology in breast cancer: From silent bystander to active facilitator. Prog Lipid Res, 69 11-20.

Christodoulatos, G. S., N. Spyrou, J. Kadillari, S. Psallida and M. Dalamaga, 2019. The Role of Adipokines in Breast Cancer: Current Evidence and Perspectives. Curr Obes Rep, 8 (4), 413-433.

Cici, D., A. Corrado, C. Rotondo, R. Colia and F. P. Cantatore, 2021. Adipokines and Chronic Rheumatic Diseases: from Inflammation to Bone Involvement. Clinical Reviews in Bone and Mineral Metabolism, 18 (4), 58-71.

Crespi, E., G. Bottai and L. Santarpia, 2016. Role of inflammation in obesity-related breast cancer. Curr Opin Pharmacol, 31 114-122.

Cymbaluk-Ploska, A., A. Chudecka-Glaz, A. Jagodzinska， E. Pius-Sadowska，A. SompolskaRzechula, B. Machalinski and J. Menkiszak, 2018. Evaluation of biologically active substances promoting the development of or protecting against endometrial cancer. Onco Targets Ther, 11 13631372.

Dalamaga, M. and G. S. Christodoulatos.2017. Visfatin, obesity, and cancer. Adipocytokines, energy balance, and cancer, Springer: 109-136.

Dalamaga, M., K. N. Diakopoulos and C. S. Mantzoros, 2012. The role of adiponectin in cancer: a review of current evidence. Endocr Rev, 33 (4), 547-594.

De Silva, S., K. H. Tennekoon and E. H. Karunanayake, 2019. Overview of the genetic basis toward early detection of breast cancer. Breast Cancer (Dove Med Press), 11 71-80.
Divella, R., R. De Luca, I. Abbate, E. Naglieri and A. Daniele, 2016. Obesity and cancer: the role of adipose tissue and adipo-cytokines-induced chronic inflammation. J Cancer, 7 (15), 2346-2359.

Dizen-Namdar, N., R. Akcilar and Z. Bayat, 2020. Association between Vaspin rs2236242 Gene Polymorphism and Psoriasis Vulgaris. Skin Pharmacol Physiol, 33 (6), 317-322.

El-Mesallamy, H. O., D. H. Kassem, E. ElDemerdash and A. I. Amin, 2011. Vaspin and visfatin/Nampt are interesting interrelated adipokines playing a role in the pathogenesis of type 2 diabetes mellitus. Metabolism, 60 (1), 6370.

El-Sagheer, G., M. Gayyed, A. Ahmad, A. Abd ElFattah and M. Mohamed, 2018. Expression of chemerin correlates with a poor prognosis in female breast cancer patients. Breast Cancer (Dove Med Press), 10, 169-176.

Fang, X., J. Wei, X. He, J. Lian, D. Han, P. An, T. Zhou, S. Liu, F. Wang and J. Min, 2018. Quantitative association between body mass index and the risk of cancer: A global Meta-analysis of prospective cohort studies. Int $\mathbf{J}$ Cancer, 143(7),1595-1603.

Fazeli, M. S., H. Dashti, S. Akbarzadeh, M. Assadi, A. Aminian, M. R. Keramati and I. Nabipour, 2013. Circulating levels of novel adipocytokines in patients with colorectal cancer. Cytokine, 62 (1), 81-85.

Feola, A., S. Ricci, S. Kouidhi, A. Rizzo, A. Penon, P. Formisano, A. Giordano, A. Di Carlo and M. Di Domenico, 2017. Multifaceted Breast Cancer: The Molecular Connection With Obesity. J Cell Physiol, 232 (1), 69-77.

Goodwin, P. J. and V. Stambolic, 2015. Impact of the obesity epidemic on cancer. Annu Rev Med, 66 281-296.

Gu, L., C. Cao, J. Fu, Q. Li, D. H. Li and M. Y. Chen, 2018. Serum adiponectin in breast cancer: A meta-analysis. Medicine (Baltimore), 97 (29), e11433.

Heiker, J. T., 2014. Vaspin (serpinA12) in obesity, insulin resistance, and inflammation. J Pept Sci, 20 (5), 299-306. 
Hida, K., J. Wada, J. Eguchi, H. Zhang, M. Baba, A. Seida, I. Hashimoto, T. Okada, A. Yasuhara, A. Nakatsuka, K. Shikata, S. Hourai, J. Futami, E. Watanabe, Y. Matsuki, R. Hiramatsu, S. Akagi, H. Makino and Y. S. Kanwar, 2005. Visceral adipose tissue-derived serine protease inhibitor: a unique insulin-sensitizing adipocytokine in obesity. Proc Natl Acad Sci U S A, 102 (30), 10610-10615.

Holman, L. L., M. Onstad, Q. Zhang, R. E. Schmandt, S. Neal, M. F. Munsell, D. Urbauer, S. Mok and K. H. Lu, 2014. Serum omentin concentration is a potential biomarker for complex atypical hyperplasia and endometrioid endometrial cancer. Gynecol Oncol, 133, 119.

Hursting, S. D., J. Digiovanni, A. J. Dannenberg, M. Azrad, D. Leroith, W. Demark-Wahnefried, M. Kakarala, A. Brodie and N. A. Berger, 2012. Obesity, energy balance, and cancer: new opportunities for prevention. Cancer Prev Res (Phila), 5 (11), 1260-1272.

Iyengar, N. M., R. Arthur, J. E. Manson, R. T. Chlebowski, C. H. Kroenke, L. Peterson, T. D. Cheng, E. C. Feliciano, D. Lane, J. Luo, R. Nassir, K. Pan, S. Wassertheil-Smoller, V. Kamensky, T. E. Rohan and A. J. Dannenberg, 2019. Association of Body Fat and Risk of Breast Cancer in Postmenopausal Women With Normal Body Mass Index: A Secondary Analysis of a Randomized Clinical Trial and Observational Study. JAMA Oncol, 5 (2), 155-163.

Iyengar, N. M., C. A. Hudis and A. J. Dannenberg, 2013. Obesity and inflammation: new insights into breast cancer development and progression. Am Soc Clin Oncol Educ Book, 46-51.

Iyengar, N. M., X. K. Zhou, A. Gucalp, P. G. Morris, L. R. Howe, D. D. Giri, M. Morrow, H. Wang, M. Pollak, L. W. Jones, C. A. Hudis and A. J. Dannenberg, 2016. Systemic Correlates of White Adipose Tissue Inflammation in Early-Stage Breast Cancer. Clin Cancer Res, 22 (9), 2283-2289.

Karabulut, S., C. U. Afsar, M. Karabulut, H. Alis, M. A. Bozkurt, F. Aydogan, M. Serilmez and F. Tas, 2016. Clinical significance of serum omentin-1 levels in patients with pancreatic adenocarcinoma. BBA Clin, 6 138-142.
Kempf, K., B. Rose, T. Illig, W. Rathmann, K. Strassburger, B. Thorand, C. Meisinger, H. E. Wichmann, C. Herder and C. Vollmert, 2010. Vaspin (SERPINA12) genotypes and risk of type 2 diabetes: Results from the MONICA/KORA studies. Exp Clin Endocrinol Diabetes, 118 (3), 184-189.

Klement, R. J. and M. K. Fink, 2016. Dietary and pharmacological modification of the insulin/IGF-1 system: exploiting the full repertoire against cancer. Oncogenesis, 5 (2), e193.

Kohan, L., A. Zarei, S. Fallahi and O. Tabiee, 2014. Association between vaspin rs2236242 gene polymorphism and polycystic ovary syndrome risk. Gene, 539 (2), 209-212.

Kojta, I., M. Chacinska and A. Blachnio-Zabielska, 2020. Obesity, Bioactive Lipids, and Adipose Tissue Inflammation in Insulin Resistance. Nutrients, 12 (5), 1305.

Laudisio, D., G. Muscogiuri, L. Barrea, S. Savastano and A. Colao, 2018. Obesity and breast cancer in premenopausal women: Current evidence and future perspectives. Eur J Obstet Gynecol Reprod Biol, 230 217-221.

Lengyel, E., L. Makowski, J. DiGiovanni and M. G. Kolonin, 2018. Cancer as a Matter of Fat: The Crosstalk between Adipose Tissue and Tumors. Trends Cancer, 4 (5), 374-384.

Leone, A., C. Nigro, A. Nicolò, I. Prevenzano, P. Formisano, F. Beguinot and C. Miele, 2021. The Dual-Role of Methylglyoxal in Tumor Progression - Novel Therapeutic Approaches. Frontiers in Oncology, 11 (822),

Li, K., L. Li, M. Yang, H. Liu, D. Liu, H. Yang, G. Boden and G. Yang, 2011. Short-term continuous subcutaneous insulin infusion decreases the plasma vaspin levels in patients with type 2 diabetes mellitus concomitant with improvement in insulin sensitivity. Eur J Endocrinol, 164 (6), 905-910. 
Lopez, R., A. Arumugam, R. Joseph, K. Monga, T. Boopalan, P. Agullo, C. Gutierrez, S. Nandy, R. Subramani, J. M. de la Rosa and R. Lakshmanaswamy, 2013. Hyperglycemia enhances the proliferation of non-tumorigenic and malignant mammary epithelial cells through increased leptin/IGF1R signaling and activation of AKT/mTOR. PLoS One, 8 (11), e79708.

Ludwig, R. G., A. L. Rocha and M. A. Mori, 2018. Circulating molecules that control brown/beige adipocyte differentiation and thermogenic capacity. Cell Biol Int, 42 (6), 701-710.

Mohanty, S. S. and P. K. Mohanty, 2021. Obesity as potential breast cancer risk factor for postmenopausal women. Genes Dis, 8 (2), 117-123.

Naeem, M., M. Hayat, S. A. Qamar, T. Mehmood, A. Munir, G. Ahmad, U. R. Azmi, M. A. Faryad, M. Z. Talib and M. Irfan, 2019. Risk factors, genetic mutations and prevention of breast cancer. Int J Biosci, 14 (4), 492-496.

Nazar, S., S. Zehra and A. Azhar, 2017. Association of single Nucleotide Missence Polymorphism Val109Asp of Omentin-1 gene and coronary artery disease in Pakistani population: Multicenter study. Pak J Med Sci, 33 (5), 1128.

Nourbakhsh, P., A. Ganji, I. Farahani, R. Hosseinian, F. Yeganefard, R. Mirzaee and G. Mosayebi, 2018. Adipokine omentin-1: A diagnostic tool in breast cancer. Int $\mathbf{J}$ Basic Sci Med, 3 (2), 89-93.

Ohashi, K., R. Shibata, T. Murohara and N. Ouchi, 2014. Role of anti-inflammatory adipokines in obesity-related diseases. Trends Endocrinol Metab, 25 (7), 348-355.

Parida, S., S. Siddharth and D. Sharma, 2019. Adiponectin, Obesity, and Cancer: Clash of the Bigwigs in Health and Disease. Int J Mol Sci, 20 (10), 2519.

Picon-Ruiz, M., C. Morata-Tarifa, J. J. ValleGoffin, E. R. Friedman and J. M. Slingerland, 2017. Obesity and adverse breast cancer risk and outcome: Mechanistic insights and strategies for intervention. CA Cancer J Clin, 67 (5), 378-397.

Reizes, O. and N. A. Berger. 2016. Adipocytokines, energy balance, and cancer, Springer.12.
Sanchez-Jimenez, F., A. Perez-Perez, L. de la Cruz-Merino and V. Sanchez-Margalet, 2019. Obesity and Breast Cancer: Role of Leptin. Front Oncol, 9596.

Schaffler, A., M. Zeitoun, H. Wobser, C. Buechler, C. Aslanidis and H. Herfarth, 2007. Frequency and significance of the novel single nucleotide missense polymorphism Val109Asp in the human gene encoding omentin in Caucasian patients with type 2 diabetes mellitus or chronic inflammatory bowel diseases. Cardiovasc Diabetol, 63.

Shen, X. D., L. Zhang, H. Che, Y. Y. Zhang, C. Yang, J. Zhou and C. Z. Liang, 2016. Circulating levels of adipocytokine omentin-1 in patients with renal cell cancer. Cytokine, 77 50-55.

Spyrou, N., K. I. Avgerinos, C. S. Mantzoros and M. Dalamaga, 2018. Classic and Novel Adipocytokines at the Intersection of Obesity and Cancer: Diagnostic and Therapeutic Strategies. Curr Obes Rep, 7 (4), 260-275.

Sun, Y. S., Z. Zhao, Z. N. Yang, F. Xu, H. J. Lu, Z. Y. Zhu, W. Shi, J. Jiang, P. P. Yao and H. P. Zhu, 2017. Risk Factors and Preventions of Breast Cancer. Int J Biol Sci, 13 (11), 1387-1397.

Tahmasebpour, N., M. A. Hosseinpour Feizi, N. Ziamajidi, N. Pouladi, V. Montazeri, M. Farhadian and R. Abbasalipourkabir, 2020. Association of Omentin-1 with Oxidative Stress and Clinical Significances in Patients with Breast Cancer. Adv Pharm Bull, 10 (1), 106-113.

Turan, H., K. O. Yaykasli, H. Soguktas, E. Yaykasli, C. Aliagaoglu, T. Erdem, M. Karkucak, E. Kaya, T. Ucgun and A. Bahadir, 2014. Omentin serum levels and omentin gene Val109Asp polymorphism in patients with psoriasis. Int $\mathbf{J}$ Dermatol, 53 (5), 601-605.

Uyeturk, U., H. Sarici, B. Kin Tekce, M. Eroglu, E. Kemahli, U. Uyeturk and A. Gucuk, 2014. Serum omentin level in patients with prostate cancer. Med Oncol, 31 (4), 923.

Wada, J., 2008. Vaspin: a novel serpin with insulin-sensitizing effects. Expert Opin Investig Drugs, 17 (3), 327-333.

Wu, Q., B. Li, Z. Li, J. Li, S. Sun and S. Sun, 2019. Cancer-associated adipocytes: key players in breast cancer progression. J Hematol Oncol, 12 (1), 95. 
Yang, R.-Z., M.-J. Lee, H. Hu, J. Pray, H.-B. Wu, B. C. Hansen, A. R. Shuldiner, S. K. Fried, J. C. McLenithan and D.-W. Gong, 2006. Identification of omentin as a novel depot-specific adipokine in human adipose tissue: possible role in modulating insulin action. Am J Physiol Endocrinol Metab, 290 (6), E1253-E1261.

Ye, J., J. Jia, S. Dong, C. Zhang, S. Yu, L. Li, C. Mao, D. Wang, J. Chen and G. Yuan, 2014. Circulating adiponectin levels and the risk of breast cancer: a meta-analysis. Eur J Cancer Prev, 23 (3), 158-165.

Yoruk, U., K. O. Yaykasli, H. Ozhan, R. Memisogullari, A. Karabacak, S. Bulur, Y. Aslantas, C. Basar and E. Kaya, 2014. Association of omentin Val109Asp polymorphism with coronary artery disease. Anadolu Kardiyol Derg, 14 (6), 511-514.
Zhang, Y.-Y. and L.-M. Zhou, 2013. Omentin-1, a new adipokine, promotes apoptosis through regulating Sirt1-dependent p53 deacetylation in hepatocellular carcinoma cells. Eur J Pharmacol, 698 (1-3), 137-144.

Zorena, K., O. Jachimowicz-Duda, D. Slezak, M. Robakowska and M. Mrugacz, 2020. Adipokines and Obesity. Potential Link to Metabolic Disorders and Chronic Complications. Int J Mol Sci, 21 (10), 3570 . 